Faraed D Salman

BDS, MSc (Assis Prof)

May Gh A1-Ajrab

BDS, MSc (Assist Lect)

\section{Natural fluoride Content of Drinking Water in Two District Areas in Iraq and Yemen (A comparative study).}

\author{
Dept of Pedod, orthod, and Prev Dentistry \\ Collage of Dentistry, University of Mosul
}

\begin{abstract}
Aims: To determine and compare the prevalence and severity of dental fluorosis in two district areas: Iraq and Yemen, in relation to age and sex for students aged 13-16 years old. Materials and methods: the study was conducted among 904 students of both countries. Five hundred and seventy two students from Iraq (Sinjar province): 334 males and 238 females. Three hundred and thirty two students: 173 males and 159 females, from Yemen (Thamar province); aged 13-16 years old were randomly selected from intermediate and secondary schools who had lived since birth in two different areas with concentration of fluoride in drinking water. Sinjar with high concentration (2.05-2.22 ppm), Thamar with moderate concentration (1.8-2.2 ppm) by the use of Dean index (1934) to assess dental fluorosis. Results: the study had shown that the prevalence of dental flourosis in Sinjar province was $52.1 \%$, $63.53 \%$ within students and teeth respectively was much higher than Thamar province which was $16.99 \%, 30.23 \%$ within students and teeth respectively; ranging from questionable, very mild forms for all age groups, while for Thamar it was ranging from very mild to moderate with significant sex difference for individuals (females reported less prevalence than males) for both provinces for the first three degrees of flourosis at $P<0.001, P \leq 0.05$. The percent age of severity for Sinjar was much higher than Thamar province which increased significantly with increasing age at $P<0.001, P<0.01$, and $P \leq$ 0.05 . Also the distribution of severity of frequency was much higher in Sinjar than Thamar with highest percentage in upper and lower posterior teeth $(75 \%, 72 \%)$ at $P<0.001$. The community flourosis index for Sinjar was 1.62 (moderate) while for Thamar province was 3.05 for these age groups (very marked). Conclusions: prevalence of dental flourosis increased infrequently with age in Sinjar while systematically in Thamar with sex difference (females reported less percentage than males).
\end{abstract}

Key words: dental flourosis, community flourosis index, natural fluoride content of drinking water.

Salman FD, AL-Ajrab MGh. Natural fluoride Content of Drinking Water in Two District Areas in Iraq and Yemen (A comparative study). Al-Rafidain Dent J. 2008; 8(1): 90-103 .

Received: $14 / 1 / 2007$

Sent to Referees: $14 / 11 / 2006$

Accepted for Publication: 10/6/2007

\section{INTRODUCTION}

Dental flourosis is a disfigurement associated with the ingestion of toxic amounts of fluoride during the period of calcification of the teeth in infancy and early childhood, the main source of flourosis is the ingestion of water containing high level of fluoride more than $1 \mathrm{ppm}$, most foods contain only very low levels of fluoride; exceptions are some fish and tea, which particularly high in fluoride may participate in fluorosis ${ }^{(1,2)}$.

Dental fluorosis, of esthetic concern is an expensive condition to treat and if left untreated can cause embarrassment for school-aged children resulting in psychological stress ${ }^{(3)}$. Fluorosed enamel has a high protein content, this results in increa-

sed porosity which in the moderate and severe forms, may eventually become stained and pitted $^{(4)}$.

The mild form of dental fluorosis is detectable only to the trained observers, while moderate and severe forms, dental fluorosis becomes a cosmetic concern specially when it averts the incisors and canines (front teeth) ${ }^{(5)}$.

The mild form of fluorosis has no effect on tooth function and may render the enamel more resistant to caries. It is not readily apparent to the affected individual or casual observer and often requires a trained specialist to detect. In contrast, 
the moderate and severe forms are generally characterized by esthetically objectionable changes in tooth color and surface irregularities.

Most investigators regard even the more advanced forms of enamel fluorosis as a cosmetic effect rather than a functional adverse effect ${ }^{(6,7)}$.

There is also mounting evidence that dental fluorosis in it's more advanced stages render the teeth more susceptible to cavities as noted by many resources ${ }^{(6-8)}$ but in general fluoride prevents tooth decay by changing the structures of enamel in infant, making it more resistant to acid attack. It also encourages the remineralization of teeth and may inhibit enzymes used by bacteria to form acid ${ }^{(9-11)}$.

The aims of the study are: To determine the prevalence of dental fluorosis in two different cities (Sinjar, and Thamar) using high and moderate levels of fluoride in drinking water and make a comparison between them. And to determine the prevalence and severity of dental fluorosis among adolescents aged (13-16) years old. As well as determination the sex difference between different age groups.

\section{MATERIALS AND METHODS}

The study was conducted in two district areas in Iraq and Yemen (Sinjar and Thamar). Sinjar province was with drinking water used, contained 2.05-2.22 ppm fluoride and the source of water is tap water from bore hole, while Thamar province was with drinking water used, contained 1.8-2.2 ppm fluoride, and the source of water is tap water from bore hole according to WHO reports ${ }^{(12)}$.

The two provinces were nearly of the same socioeconomic and educational status. The population of the study was students (girls and boys) aged (13-16) years. They had been examined for estimation the prevalence of dental fluorosis, then compared between the two provinces. The students that had been examined were in the secondary schools, and the selection of schools was randomly, two secondary schools for boys and two secondary schools for girls had been selected for each province and the classes selected are from first up to fourth intermediate classes. All the students examined had lived continuously from birth in the district areas which they had been examined ${ }^{(12)}$.

The students had been examined in a suitable room under natural day light using plane mouth mirrors, sharp sickle shaped caries explorers with the students sitting in a chair in front of the examiner. Any student that didn't born or live in those areas had been excluded from the examination. Also informations regarding name, age and sex of the student were registered prior to the examination on a special form which contained the assessment of dental fluorosis index. Also the student was asked about using any form of fluoride supplements and if the answer was yes, the student was excluded from the study.

\section{Oral examination:}

All the examined teeth were dried with cotton wool, the tooth was considered a fully erupted when at least $2 / 3$ of the crown erupted with no gingiva covering it (i.e. the examination included all fully erupted permanent teeth and all primary teeth were excluded from the examination also permanent teeth with crown or labial veneer or retained root were excluded from the examination.

The diagnosis of dental fluorosis was recorded according to the criteria of Dean index ${ }^{(13)}$. Each tooth had been graded as normal or one of the following degrees of fluorosis (questionable, very mild, mild, moderate and severe) for assessment of prevalence of dental fluorosis within individual and teeth.

In addition to assessing the degree of dental fluorosis within individual, Dean devised means of calculating the degree of fluorosis within a community by the use of community fluorosis index ${ }^{(14,15)}$.

Community fluorosis index $=\Sigma$ no. of individual $\times \frac{\text { Statistical weight }}{\text { Total no. of individuals examined }}$

Data were analyzed using numbers of students teeth and percentages. Z-test was used for determining sex differences for all forms of dental fluorosis. 


\section{RESULTS}

The distribution of the sample by sex, age and area was shown in Table (1). The total sample was divided into two main groups. The first group consisted of 572 (63.27\%) students lived continuously from birth in high level natural fluoride area (Sinjar province) and the second consisted of $332(36.72 \%)$ students lived continuously from birth in the moderate natural fluoride area (Thamar province). The total sample consisted of 904 students 507 males $(56.1 \%)$ and 397 (43.9\%) females.

Table (2) showed, the number and percentage of students who suffer from dental fluorosis in Sinjar province. The results indicated that $47.9 \%$ of individuals have normal teeth which means that $52.1 \%$ of individuals had dental fluorosis. The percentage of normal individuals increased with increasing age of the students with significant difference between females for the age groups 13, 14 years old according to Z-test. The total females reported higher normal percentage than total males at $P<0.01$. The results showed that questionable, very mild, mild forms of dental fluorosis had nearly the same percentages in all age groups and for the total sample. The results revealed that the severity of dental fluorosis decreased with increasing age of the students specially for very mild from with significant difference between age groups at $P \leq 0.05$. The total males reported more severe dental fluorosis for the mild form with significant difference at $P<0.01$ using Z-test.

Table (3) showed, the number and the percentage of students who suffer from dental fluorosis in Thamar province. The results indicated that $83.1 \%$ of individuals have normal teeth, which means that $16.99 \%$ of the individuals have dental fluorosis. The percentage of normal individuals decreased with increasing age of the students. The results indicated that, the very mild and moderate forms of dental fluorosis had the higher percentages in all age groups and for the total sample. The results revealed that, the very mild form of fluorosis has greater severity with increasing age of students. The total males reported more severe dental fluorosis (moderate and severe) with no significant difference between age groups according to $\mathrm{Z}$-test.

Table (4) described that, the number and percentage of Sinjar students teeth distributed according to Dean index of fluorosis by age and sex. The results showed that the questionable form of dental fluorosis had a higher percentage for the total sample (23.83\%) and for all age groups followed by others forms of dental fluorosis as decreased the percentage with the increase of the severity.

The study revealed that the severity for the mild, moderate, severe forms of dental fluorosis increased with age. The total males reported more severe dental fluorosis than total females in categories very mild, mild, moderate and severe forms and at $P \leq 0.01$ for very mild and moderate forms, and at $P \leq 0.001$ for mild and severe forms, while for total females reported higher percentage than total males with significant difference at $P \leq 0.05$.

Table (1): Distribution of sample according to age, sex and area.

\begin{tabular}{cccccccc}
\hline \multirow{2}{*}{ Area } & \multirow{2}{*}{ Age (year) } & \multicolumn{2}{c}{ Males } & \multicolumn{2}{c}{ Females } & \multicolumn{2}{c}{ Total } \\
\cline { 3 - 8 } & & No. & $\%$ & No. & \% & No. & \% \\
\hline \multirow{4}{*}{ Sinjar } & 13 & 107 & 64.1 & 60 & 35.9 & 167 & 29.2 \\
& 14 & 93 & 54.7 & 77 & 45.3 & 170 & 29.7 \\
& 15 & 73 & 57.5 & 54 & 42.5 & 127 & 22.2 \\
& 16 & 61 & 56.5 & 47 & 43.5 & 108 & 18.9 \\
\hline \multicolumn{2}{c}{ Total } & 334 & 58.4 & 238 & 41.6 & 572 & 100 \\
\multirow{3}{*}{ Yemen } & 13 & 44 & 60.3 & 29 & 39.7 & 73 & 22.0 \\
& 14 & 41 & 47.7 & 45 & 52.3 & 86 & 25.9 \\
& 15 & 44 & 51.2 & 42 & 48.8 & 86 & 25.9 \\
& 16 & 44 & 50.6 & 43 & 49.4 & 87 & 26.2 \\
\hline \multicolumn{2}{c}{ Total } & 173 & 52.1 & 159 & 47.9 & 332 & 100 \\
\hline \multicolumn{2}{c}{ Total sample } & 507 & 56.1 & 397 & 43.9 & 904 & 100 \\
\hline
\end{tabular}

No.= Number; $\%=$ Percentage 
Table (2): Number and percentage of Sinjar students distributed according to Dean index of florosis by age and sex ${ }^{(13)}$.

\begin{tabular}{|c|c|c|c|c|c|c|c|c|c|c|c|c|c|c|}
\hline \multirow{3}{*}{$\begin{array}{c}\text { Age } \\
\text { (year) }\end{array}$} & \multirow{3}{*}{ Sex } & \multirow{3}{*}{ No. } & \multicolumn{12}{|c|}{ Dean index } \\
\hline & & & \multicolumn{2}{|c|}{ Normal } & \multicolumn{2}{|c|}{ Questionable } & \multicolumn{2}{|c|}{ Very mild } & \multicolumn{2}{|c|}{ Mild } & \multicolumn{2}{|c|}{ Moderate } & \multicolumn{2}{|c|}{ Severe } \\
\hline & & & No. & $\%$ & No. & $\%$ & No & $\%$ & No. & $\%$ & No. & $\%$ & No & $\%$ \\
\hline \multirow{2}{*}{13} & $\mathbf{M}$ & 107 & 39 & 36.4 & 24 & 22.4 & 25 & 23.4 & 19 & 17.8 & 0 & 0.0 & 0 & 0.0 \\
\hline & $\mathbf{F}$ & 60 & 33 & $55.0 *$ & 11 & 18.3 & 11 & 18.3 & 5 & 8.3 & 0 & 0.0 & 0 & 0.0 \\
\hline \multicolumn{2}{|c|}{ Total } & 167 & 72 & 43.1 & 35 & 21.0 & 36 & $21.6 \mathrm{a}$ & 24 & 14.4 & 0 & 0.0 & 0 & 0.0 \\
\hline \multirow{2}{*}{14} & $\mathbf{M}$ & 93 & 41 & 44.1 & 14 & 15.1 & 17 & 18.3 & 20 & 21.5 & 1 & 1.1 & 0 & 0.0 \\
\hline & $\mathbf{F}$ & 77 & 46 & $59.7 *$ & 17 & 22.1 & 9 & 11.7 & 5 & $6.5^{* *}$ & 0 & 0.0 & 0 & 0.0 \\
\hline \multicolumn{2}{|c|}{ Total } & 170 & 87 & 51.2 & 31 & 18.2 & 26 & $15.3 \mathrm{a}$ & 25 & 14.7 & 1 & 0.6 & 0 & 0.0 \\
\hline \multirow{2}{*}{15} & $\mathbf{M}$ & 73 & 31 & 42.5 & 12 & 16.4 & 11 & 15.1 & 17 & 23.3 & 2 & 2.7 & 0 & 0.0 \\
\hline & $\mathbf{F}$ & 54 & 31 & 57.4 & 9 & 16.7 & 7 & 13.0 & 7 & 13.0 & 0 & 0.0 & 0 & 0.0 \\
\hline \multicolumn{2}{|c|}{ Total } & 127 & 62 & 48.8 & 21 & 16.5 & 18 & $14.2 \mathrm{ab}$ & 24 & 18.9 & 2 & 1.6 & 0 & 0.0 \\
\hline \multirow{2}{*}{16} & $\mathbf{M}$ & 61 & 32 & 52.5 & 10 & 16.4 & 3 & 4.9 & 15 & 24.6 & 1 & 1.6 & 0 & 0.0 \\
\hline & $\mathbf{F}$ & 47 & 21 & 44.7 & 11 & 23.4 & 4 & 8.5 & 9 & 19.1 & 1 & 2.1 & 1 & 2.1 \\
\hline \multicolumn{2}{|c|}{ Total } & 108 & 53 & 49.1 & 21 & 19.4 & 7 & $6.5 b$ & 24 & 22.2 & 2 & 1.9 & 1 & 0.9 \\
\hline \multirow{2}{*}{\multicolumn{2}{|c|}{ Total males }} & 334 & 143 & 42.8 & 60 & 18.0 & 56 & 16.8 & 71 & 21.3 & 4 & 1.2 & 0 & 0.0 \\
\hline \multirow{2}{*}{\multicolumn{2}{|c|}{ Total sample }} & 238 & 131 & $55.0 * *$ & 48 & 20.2 & 31 & 13.0 & 26 & $10.9 * *$ & 1 & 0.4 & 1 & 0.4 \\
\hline & & 572 & 274 & 47.9 & 108 & 18.9 & 87 & 15.2 & 97 & 17.0 & 5 & 0.9 & 1 & 0.2 \\
\hline
\end{tabular}

Table (3): Number and percentage of Thamar students distributed according to Dean index of florosis by age and sex ${ }^{(13)}$.

\begin{tabular}{|c|c|c|c|c|c|c|c|c|c|c|c|c|c|c|}
\hline \multirow{3}{*}{$\begin{array}{c}\text { Age } \\
\text { (year) }\end{array}$} & \multirow{3}{*}{ Sex } & \multirow{3}{*}{ No. } & \multicolumn{12}{|c|}{ Dean index* } \\
\hline & & & \multicolumn{2}{|c|}{ Normal } & \multicolumn{2}{|c|}{ Questionable } & \multicolumn{2}{|c|}{ Very mild } & \multicolumn{2}{|c|}{ Mild } & \multicolumn{2}{|c|}{ Moderate } & \multicolumn{2}{|c|}{ Severe } \\
\hline & & & No. & $\%$ & No. & $\%$ & No & $\%$ & No. & $\%$ & No. & $\%$ & No & $\%$ \\
\hline \multirow{2}{*}{13} & M & 44 & 41 & 93.2 & 0 & 0.0 & 1 & 2.3 & 2 & 4.5 & 0 & 0.0 & 0 & 0.0 \\
\hline & $\mathbf{F}$ & 29 & 26 & 89.7 & 0 & 0.0 & 1 & 3.4 & 0 & 0.0 & 1 & 3.4 & 1 & 3.4 \\
\hline \multicolumn{2}{|c|}{ Total } & 73 & 67 & 91.8 & 0 & 0.0 & 2 & 2.7 & 2 & 2.7 & 1 & 1.4 & 1 & 1.4 \\
\hline \multirow{2}{*}{14} & $\mathbf{M}$ & 41 & 35 & 85.4 & 0 & 0.0 & 2 & 4.9 & 0 & 0.0 & 1 & 2.4 & 3 & 7.3 \\
\hline & $\mathbf{F}$ & 45 & 37 & 82.2 & 0 & 0.0 & 3 & 6.7 & 0 & 0.0 & 3 & 6.7 & 2 & 4.4 \\
\hline \multicolumn{2}{|c|}{ Total } & 86 & 72 & 83.7 & 0 & 0.0 & 5 & 5.8 & 0 & 0.0 & 4 & 4.7 & 5 & 5.8 \\
\hline \multirow{2}{*}{15} & $\mathbf{M}$ & 44 & 34 & 77.3 & 0 & 0.0 & 3 & 6.8 & 0 & 0.0 & 5 & 11.4 & 2 & 4.5 \\
\hline & $\mathbf{F}$ & 42 & 34 & 81.0 & 0 & 0.0 & 3 & 7.1 & 3 & 7.1 & 2 & 4.8 & 0 & 0.0 \\
\hline \multicolumn{2}{|c|}{ Total } & 86 & 68 & 79.1 & 0 & 0.0 & 6 & 7.0 & 3 & 3.5 & 7 & 8.1 & 2 & 2.3 \\
\hline \multirow{2}{*}{16} & $\mathbf{M}$ & 44 & 36 & 81.6 & 2 & 4.5 & 5 & 11.4 & 0 & 0.0 & 1 & 2.3 & 0 & 0.0 \\
\hline & $\mathbf{F}$ & 43 & 33 & 76.7 & 2 & 4.7 & 8 & 18.6 & 0 & 0.0 & 0 & 0.0 & 0 & 0.0 \\
\hline \multicolumn{2}{|c|}{ Total } & 87 & 69 & 79.3 & 4 & 4.6 & 13 & 14.9 & 0 & 0.0 & 1 & 1.1 & 0 & 0.0 \\
\hline \multicolumn{2}{|c|}{ Total males } & 173 & 146 & 84.4 & 2 & 1.2 & 11 & 6.4 & 2 & 1.2 & 7 & 4.0 & 5 & 2.9 \\
\hline \multicolumn{2}{|c|}{ Total females } & 159 & 130 & 81.8 & 2 & 1.3 & 15 & 9.4 & 3 & 1.9 & 6 & 3.8 & 3 & 1.9 \\
\hline \multicolumn{2}{|c|}{ Total sample } & 332 & 276 & 83.1 & 4 & 1.2 & 26 & 7.8 & 5 & 1.5 & 13 & 3.9 & 8 & 2.4 \\
\hline
\end{tabular}

*No significant difference between males and females and among age groups according to Z-test; No.= Number; $\%=$ Percentage; $\mathrm{M}=$ mail $; \mathrm{F}=$ female 
Table (4): Number and percentage of Sinjar student's teeth distributed according to Dean index of fluorosis by age and sex ${ }^{(13)}$.

\begin{tabular}{|c|c|c|c|c|c|c|c|c|c|c|c|c|c|c|}
\hline \multirow{3}{*}{$\begin{array}{c}\text { Age } \\
\text { (year) }\end{array}$} & \multirow{3}{*}{ Sex } & \multirow{3}{*}{ No. } & \multicolumn{12}{|c|}{ Dean index } \\
\hline & & & \multicolumn{2}{|c|}{ Normal } & \multicolumn{2}{|c|}{ Questionable } & \multicolumn{2}{|c|}{ Very mild } & \multicolumn{2}{|c|}{ Mild } & \multicolumn{2}{|c|}{ Moderate } & \multicolumn{2}{|c|}{ Severe } \\
\hline & & & No. & $\%$ & No. & $\%$ & No & $\%$ & No. & $\%$ & No. & $\%$ & No. & $\%$ \\
\hline \multirow[b]{2}{*}{13} & $\mathbf{M}$ & 107 & 1114 & 37.18 & 712 & 23.77 & 623 & 20.79 & 417 & 13.92 & 58 & 1.94 & 72 & 2.4 \\
\hline & $\mathbf{F}$ & 60 & 732 & $43.57^{\S}$ & 414 & 24.64 & 341 & 20.30 & 164 & $9.76^{\S}$ & 23 & 1.37 & 6 & $0.36^{\S}$ \\
\hline \multicolumn{2}{|c|}{ Total } & 167 & 1846 & 39.48 & 1126 & 24.08 & 964 & 20.62 & 581 & 12.43 & 81 & 1.73 & 78 & 1.67 \\
\hline \multirow{2}{*}{14} & $\mathbf{M}$ & 93 & 927 & 35.6 & 624 & 23.96 & 513 & 19.70 & 411 & 15.78 & 89 & 3.42 & 40 & 1.54 \\
\hline & $\mathbf{F}$ & 77 & 1004 & $46.57^{\S}$ & 539 & 25.00 & 334 & $15.49^{\S}$ & 198 & $9.18^{\S}$ & 64 & $2.97^{¥}$ & 17 & 0.79 \\
\hline \multicolumn{2}{|c|}{ Total } & 170 & 1931 & 40.57 & 1163 & 24.43 & 847 & 1779 & 609 & 12.79 & 153 & 3.21 & 57 & 1.2 \\
\hline \multirow{2}{*}{15} & M & 73 & 662 & 32.39 & 484 & 23.68 & 405 & 19.81 & 365 & 17.86 & 90 & 4.4 & 38 & 1.86 \\
\hline & $\mathbf{F}$ & 54 & 641 & $42.39^{\S}$ & 377 & 24.93 & 245 & $16.2^{¥}$ & 202 & $13.36^{\S}$ & 36 & 2.38 & 11 & 0.73 \\
\hline \multicolumn{2}{|c|}{ Total } & 127 & 1303 & 36.64 & 861 & 24.21 & 650 & 18.28 & 567 & 15.94 & 126 & 3.54 & 49 & 1.38 \\
\hline \multirow{2}{*}{16} & $\mathbf{M}$ & 61 & 741 & 43.38 & 355 & 20.78 & 253 & 14.81 & 267 & 15.63 & 70 & 4.1 & 22 & 1.29 \\
\hline & $\mathbf{F}$ & 47 & 500 & $37.99^{\S}$ & 311 & 23.63 & 242 & $18.39^{¥}$ & 181 & 13.75 & 47 & 3.57 & 35 & $2.66^{\ddagger}$ \\
\hline \multicolumn{2}{|c|}{ Total } & 108 & 1241 & 41.04 & 666 & 22.02 & 495 & 16.37 & 448 & 14.81 & 117 & 3.87 & 57 & 1.88 \\
\hline \multicolumn{2}{|c|}{ Total males } & 334 & 3444 & 36.83 & 2175 & 23.26 & 1794 & 19.18 & 1460 & 15.61 & 307 & 3.28 & 172 & 1.84 \\
\hline \multicolumn{2}{|c|}{ Total females } & 238 & 2877 & $43.17^{\S}$ & 1641 & $24.62 *$ & 1162 & $17.44^{¥}$ & 745 & $11.18^{\S}$ & 170 & $2.55^{¥}$ & 69 & $1.04^{\S}$ \\
\hline \multicolumn{2}{|c|}{ Total sample } & 572 & 6321 & 39.47 & 3816 & 23.83 & 2956 & 18.46 & 2205 & 13.77 & 477 & 2.98 & 241 & 1.5 \\
\hline
\end{tabular}

In Table (5): The number and percentage of Thamar student's teeth distributed according to Dean index of fluorosis by age and sex. The study reported that $69.77 \%$ of all teeth in Thamar province is normal. On the other hand, only $30.23 \%$ of teeth is affected by dental fluorosis. The higher percentage for at the total sample was for very mild form (12.99\%) followed by moderate form $(5.5 \%)$.

The total males reported more severe dental fluorosis than females (moderate and severe forms) with significant difference at $P \leq 0.05$ and at $P<0.001$, while for very mild and mild forms the opposite figure is true at $P<0.001$.

Table (6) reported, the number of individuals in Sinjar province distributed according to Dean index categories from 0 to 4 . The table showed the statistical weight of each category. Community fluorosis index for Sinjar $=572 / 354=1.62$

Table (7) showed, the number of individuals in Thamar province distributed according to Dean index categories from 0 to 4 . Community fluorosis index for Thamar $=332 / 109=3.05$

In Table (8): The number and percentage of Iraqi and Yemeni students distributed according to degree of severity of fluorosis by sex for 13 years old.

There is significant difference in total between normal individuals (both province) at $P<0.001$, and there was significant difference for questionable, very mild, mild form at $P<0.001, P<0.01$ respectively.

The percentage of individuals decreesed with increase severity of fluorosis in both Thamar and Sinjar.

The number and percentage of Iraqi and Yemeni students distributed according to degree of severity of fluorosis by sex for 14 years old. There was significant difference in total between normal individuals (both provinces) at $P<0.001$ and there was significant difference for questionable, very mild, mild forms at $P<0.001$, $P \leq 0.05, P<0.001$ respectively, at Table (9). The percentage of individuals decreeased with increase severity in Thamar and Sinjar.

Table (10) showed number and percentage of Iraqi and Yemeni students distributed according to Dean index of fluorosis by sex for 15 years old.

There was significant difference in total between normal individuals (both 
provinces) at $P<0.001$ and there was significant difference for questionable, mild and moderate forms at $P<0.001, P \leq$ 0.05 respectively.

Table (11) described the number and percentage of Iraqi and Yemeni students distributed according to Dean index of fluorosis by sex for 16 years old.

There was significant difference in total between normal individuals (both province) at $P<0.001$, and there was significant difference for questionable, mild forms at $P<0.01, P<0.001$ respectively.
The percentage of normal individuals in total in Thamar decreased with increasing age while in Sinjar it was infrequent.

The percentage of severity of fluorosis in Sinjar is higher than in Thamar in all age groups for the first two age groups: Questionable, very mild, mild. For 15 years: Questionable, mild and moderate. And for 16 years: Questionable and mild.

The questionable form in Yemen is 0 in the first three age groups but increased significantly to 4.6 in 16 years old.

Table (5): Number and percentage of Thamar student's teeth distributed according to Dean index of fluorosis by age and sex ${ }^{(13)}$.

\begin{tabular}{|c|c|c|c|c|c|c|c|c|c|c|c|c|c|c|}
\hline \multirow{3}{*}{$\begin{array}{c}\text { Age } \\
\text { (year) }\end{array}$} & \multirow{3}{*}{ Sex } & \multirow{3}{*}{ No. } & \multicolumn{12}{|c|}{ Dean index } \\
\hline & & & \multicolumn{2}{|c|}{ Normal } & \multicolumn{2}{|c|}{$\begin{array}{c}\text { Questionabl } \\
\text { e }\end{array}$} & \multicolumn{2}{|c|}{ Very mild } & \multicolumn{2}{|c|}{ Mild } & \multicolumn{2}{|c|}{ Moderate } & \multicolumn{2}{|c|}{ Severe } \\
\hline & & & No. & $\%$ & No. & $\%$ & No. & $\%$ & No. & $\%$ & No. & $\%$ & No & $\%$ \\
\hline \multirow{2}{*}{13} & M & 44 & 950 & 77.11 & 33 & 2.68 & 17 & 9.5 & 79 & 6.41 & 43 & 3.49 & 10 & 0.81 \\
\hline & $\mathbf{F}$ & 29 & 602 & 74.14 & 5 & $0.62^{\S}$ & 95 & 11.7 & 40 & 4.93 & 40 & 4.93 & 30 & $3.69^{\S}$ \\
\hline \multicolumn{2}{|c|}{ Total } & 73 & 1552 & 75.93 & 38 & 1.86 & 212 & 10.37 & 119 & 5.82 & 83 & 4.06 & 40 & 1.96 \\
\hline \multirow{2}{*}{14} & $\mathbf{M}$ & 41 & 852 & 74.22 & 17 & 1.84 & 102 & 8.89 & 31 & 2.7 & 46 & 4.01 & 100 & 8.71 \\
\hline & $\mathbf{F}$ & 45 & 875 & $69.44^{¥}$ & 14 & 1.11 & 148 & $11.75^{*}$ & 5.8 & $4.6^{*}$ & 107 & $8.49^{\S}$ & 58 & $4.6^{\S}$ \\
\hline \multicolumn{2}{|c|}{ Total } & 86 & 1727 & 71.72 & 31 & 1.29 & 250 & 10.38 & 89 & 3.7 & 153 & 6.35 & 158 & 6.56 \\
\hline \multirow{2}{*}{15} & $\mathbf{M}$ & 44 & 808 & 65.58 & 18 & 1.46 & 133 & 10.8 & 31 & 2.52 & 169 & 13.72 & 73 & 5.93 \\
\hline & $\mathbf{F}$ & 42 & 752 & 63.95 & 24 & 2.04 & 203 & $17.26^{\S}$ & 114 & $9.69^{\S}$ & 71 & $6.04^{\S}$ & 12 & 1.02 \\
\hline \multicolumn{2}{|c|}{ Total } & 86 & 1560 & 64.78 & 42 & 1.74 & 336 & 13.95 & 145 & 6.02 & 240 & 9.97 & 85 & 3.53 \\
\hline \multirow{2}{*}{16} & M & 44 & 836 & 67.87 & 123 & 9.98 & 203 & 16.48 & 30 & 2.44 & 31 & 2.52 & 9 & 0.73 \\
\hline & $\mathbf{F}$ & 43 & 811 & 67.36 & 159 & $13.21 *$ & 207 & 17.19 & 17 & 1.41 & 4 & $0.33^{\S}$ & 6 & 0.5 \\
\hline \multicolumn{2}{|c|}{ Total } & 87 & 1647 & 67.61 & 282 & 11.58 & 410 & 16.83 & 47 & 1.93 & 35 & 1.44 & 15 & 0.62 \\
\hline \multicolumn{2}{|c|}{ Total males } & 173 & 3446 & $71.14^{¥}$ & 191 & 3.94 & 555 & 11.46 & 171 & 3.53 & 289 & 5.97 & 192 & 3.96 \\
\hline \multicolumn{2}{|c|}{ Total females } & 159 & 3040 & 68.28 & 202 & 4.54 & 653 & $14.67^{\S}$ & 229 & $5.14^{\S}$ & 222 & $4.99 *$ & 106 & $2.38^{\S}$ \\
\hline \multicolumn{2}{|c|}{ Total sample } & 332 & 6486 & 69.77 & 393 & 4.23 & 1208 & 12.99 & 400 & 4.3 & 511 & 5.5 & 298 & 3.21 \\
\hline
\end{tabular}

Table (6): Number of percentage for each category of Dean index and its statistical weight for Sinjar province.

\begin{tabular}{ccc}
\hline Persons' number & Score & Statistical weight \\
\hline 274.00 & 0 & 0.00 \\
108.00 & 0.5 & 54.00 \\
87.00 & 1 & 87.00 \\
97.00 & 2 & 194.00 \\
5.00 & 3 & 15.00 \\
1.00 & 4 & 4.00 \\
\hline Total 572.00 & & Total 354.00
\end{tabular}

Community fluorosis index $=572 / 354=1.62$ 
Table (7): Number of percentage for each category of Dean index and its statistical weight for Thamar province.

\begin{tabular}{ccc}
\hline Persons' number & Score & Statistical weight \\
\hline 276.00 & 0 & 0.00 \\
4.00 & 0.5 & 2.00 \\
26.00 & 1 & 26.00 \\
5.00 & 2 & 10.00 \\
13.00 & 3 & 39.00 \\
8.00 & 4 & 32.00 \\
\hline Total 332.00 & & Total 109.00 \\
\hline
\end{tabular}

Community fluorosis index $=332 / 109=3.05$

Table (8): Number and percentage of Iraqi and Yemeni students distributed according to Dean index of fluorosis by sex for 13 years old ${ }^{(13)}$.

\begin{tabular}{|c|c|c|c|c|c|c|c|c|c|c|c|c|}
\hline \multirow{3}{*}{ Dean index } & \multicolumn{4}{|c|}{ Males } & \multicolumn{4}{|c|}{ Females } & \multicolumn{4}{|c|}{ Total } \\
\hline & \multicolumn{2}{|c|}{ Sinjar } & \multicolumn{2}{|c|}{ Yemen } & \multicolumn{2}{|c|}{ Sinjar } & \multicolumn{2}{|c|}{ Yemen } & \multicolumn{2}{|c|}{ Sinjar } & \multicolumn{2}{|c|}{ Yemen } \\
\hline & No. & $\%$ & No. & $\%$ & No. & $\%$ & No. & $\%$ & No. & $\%$ & No. & $\%$ \\
\hline Normal & 39 & 36.4 & 41 & $93.2^{\S}$ & 33 & 55 & 26 & $89.7^{7}$ & 72 & 43.1 & 67 & $91.8^{\S}$ \\
\hline Questionable & 24 & 22.4 & 0 & $0.0^{\S}$ & 11 & 18.3 & 0 & $0.0^{*}$ & 35 & 21.0 & 0 & $0.0^{\S}$ \\
\hline Very mild & 25 & 23.4 & 1 & $2.3^{¥}$ & 11 & 18.3 & 1 & 3.4 & 36 & 21.6 & 2 & $2.7^{\S}$ \\
\hline Mild & 19 & 17.8 & 2 & $4.5^{*}$ & 5 & 8.3 & 0 & 0.0 & 24 & 14.4 & 2 & $2.7^{¥}$ \\
\hline Moderate & 0 & 0.0 & 0. & 0.0 & 0 & 0.0 & 1 & 3.4 & 0 & 0.0 & 1 & 1.4 \\
\hline Severe & 0 & 0.0 & 0 & 0.0 & 0 & 0.0 & 1 & 3.4 & 0 & 0.0 & 1 & 1.4 \\
\hline Total & 107 & 100 & 44 & 100 & 60 & 100 & 29 & 100 & 167 & 100 & 73 & 100 \\
\hline
\end{tabular}

Table (9): Number and percentage of Iraqi and Yemeni students distributed according to Dean index of fluorosis by sex for 14 years old ${ }^{(13)}$.

\begin{tabular}{|c|c|c|c|c|c|c|c|c|c|c|c|c|}
\hline \multirow{3}{*}{ Dean index } & \multicolumn{4}{|c|}{ Males } & \multicolumn{4}{|c|}{ Females } & \multicolumn{4}{|c|}{ Total } \\
\hline & \multicolumn{2}{|c|}{ Sinjar } & \multicolumn{2}{|c|}{ Yemen } & \multicolumn{2}{|c|}{ Sinjar } & \multicolumn{2}{|c|}{ Yemen } & \multicolumn{2}{|c|}{ Sinjar } & \multicolumn{2}{|c|}{ Yemen } \\
\hline & No. & $\%$ & No. & $\%$ & No. & $\%$ & No. & $\%$ & No. & $\%$ & No. & $\%$ \\
\hline Normal & 41 & 44.1 & 35 & $85.4^{\S}$ & 46 & 59.7 & 37 & $82.2 *$ & 87 & 51.2 & 72 & $83.7^{\S}$ \\
\hline Questionable & 14 & 15.1 & 0 & $0.00^{\ddagger}$ & 17 & 22.1 & 0 & $0.0^{\S}$ & 31 & 18.2 & 0 & $0.0^{\S}$ \\
\hline Very mild & 17 & 18.3 & 2 & $4.9^{*}$ & 9 & 11.7 & 3 & 6.7 & 26 & 15.3 & 5 & $5.8^{*}$ \\
\hline Mild & 20 & 21.5 & 0 & $0.0^{¥}$ & 5 & 6.5 & 0 & 0.0 & 25 & 14.7 & 0 & $0.0^{\S}$ \\
\hline Moderate & 1 & 1.1 & 1 & 2.4 & 0 & 0.0 & 3 & 6.7 & 1 & 0.6 & 4 & 4.7 \\
\hline Severe & 0 & 0.0 & 3 & 7.3 & 0 & 0.0 & 2 & 4.4 & 0 & 0.0 & 5 & 5.8 \\
\hline Total & 93 & 100 & 41 & 100 & 77 & 100 & 45 & 100 & 170 & 100 & 86 & 100 \\
\hline
\end{tabular}

* Significant difference between Sinjar and Yemen at $P \leq 0.05,{ }^{¥}$ at $P<0.01$ and ${ }^{\S}$ at $P<0.001$; No.= Number; $\%=$ Percentage. 
Salman FD, AL-Ajrab MGh.

Table (10): Number and percentage of Iraqi and Yemeni students distributed according to Dean index of fluorosis by sex for 15 years old ${ }^{(13)}$.

\begin{tabular}{|c|c|c|c|c|c|c|c|c|c|c|c|c|}
\hline \multirow{3}{*}{ Dean index } & \multicolumn{4}{|c|}{ Males } & \multicolumn{4}{|c|}{ Females } & \multicolumn{4}{|c|}{ Total } \\
\hline & \multicolumn{2}{|c|}{ Sinjar } & \multicolumn{2}{|c|}{ Yemen } & \multicolumn{2}{|c|}{ Sinjar } & \multicolumn{2}{|c|}{ Yemen } & \multicolumn{2}{|c|}{ Sinjar } & \multicolumn{2}{|c|}{ Yemen } \\
\hline & No. & $\%$ & No. & $\%$ & No. & $\%$ & No. & $\%$ & No. & $\%$ & No. & $\%$ \\
\hline Normal & 31 & 42.5 & 34 & $77.3^{\S}$ & 31 & 57.4 & 34 & $81.0^{*}$ & 62 & 48.8 & 68 & $79.1^{\S}$ \\
\hline Questionable & 12 & 16.4 & 0 & $0.0^{¥}$ & 9 & 16.7 & 0 & $0.0^{¥}$ & 21 & 16.5 & 0 & $0.0^{\S}$ \\
\hline Very mild & 11 & 15.1 & 3 & $6.8^{\S}$ & 7 & 13.0 & 3 & 7.1 & 18 & 14.2 & 6 & 7.0 \\
\hline Mild & 17 & 23.3 & 0 & $0.0^{\S}$ & 7 & 13.0 & 3 & 7.1 & 24 & 18.9 & 3 & $3.5^{\S}$ \\
\hline Moderate & 2 & 2.7 & 5 & 11.4 & 0 & 0.0 & 2 & 4.8 & 2 & 1.6 & 7 & $8.1 *$ \\
\hline Severe & 0 & 0.0 & 2 & 4.5 & 0 & 0.0 & 0 & 0.0 & 0 & 0.0 & 2 & 2.3 \\
\hline Total & 73 & 100 & 44 & 100 & 54 & 100 & 42 & 100 & 127 & 100 & 86 & 100 \\
\hline
\end{tabular}

Significant difference between Sinjar and Yemen at $P \leq 0.05,{ }^{¥}$ at $P<0.01$ and ${ }^{\S}$ at $P<0.001 ;$ No.= Number; $\%=$ Percentage.

Table (11): Number and percentage of Iraqi and Yemeni students distributed according to Dean index of fluorosis by sex for 16 years old ${ }^{(13)}$.

\begin{tabular}{|c|c|c|c|c|c|c|c|c|c|c|c|c|}
\hline \multirow{3}{*}{ Dean index } & \multicolumn{4}{|c|}{ Males } & \multicolumn{4}{|c|}{ Females } & \multicolumn{4}{|c|}{ Total } \\
\hline & \multicolumn{2}{|c|}{ Sinjar } & \multicolumn{2}{|c|}{ Yemen } & \multicolumn{2}{|c|}{ Sinjar } & \multicolumn{2}{|c|}{ Yemen } & \multicolumn{2}{|c|}{ Sinjar } & \multicolumn{2}{|c|}{ Yemen } \\
\hline & No. & $\%$ & No. & $\%$ & No. & $\%$ & No. & $\%$ & No. & $\%$ & No. & $\%$ \\
\hline Normal & 32 & 52.5 & 36 & $81.6^{¥}$ & 21 & 44.7 & 33 & $76.7^{¥}$ & 53 & 49.1 & 69 & $79.3^{\S}$ \\
\hline Questionable & 10 & 16.4 & 2 & 4.5 & 11 & 23.4 & 2 & $4.7^{*}$ & 21 & 19.4 & 4 & $4.6^{¥}$ \\
\hline Very mild & 3 & 4.9 & 5 & 11.4 & 4 & 8.5 & 8 & 18.6 & 7 & 6.5 & 13 & 14.9 \\
\hline Mild & 15 & 24.6 & 0 & $0.0^{\S}$ & 9 & 19.1 & 0 & $0.0^{¥}$ & 24 & 22.2 & 0 & $0.0^{\S}$ \\
\hline Moderate & 1 & 1.6 & 1 & 2.3 & 1 & 2.1 & 0 & 0.0 & 2 & 1.9 & 1 & 1.1 \\
\hline Severe & 0 & 0.0 & 0 & 0.0 & 1 & 2.1 & 0 & 0.0 & 1 & 0.9 & 0 & 0.0 \\
\hline Total & 61 & 100 & 44 & 100 & 47 & 100 & 43 & 100 & 108 & 100 & 87 & 100 \\
\hline
\end{tabular}

Significant difference between Sinjar and Yemen at $P \leq 0.05,{ }^{\ddagger}$ at $P<0.01$ and ${ }^{\S}$ at $P<0.001$; No.= Number; $\%=$ Percentage.

Figure (1): Showed frequency distribution of severity of dental fluorosis for upper anterior teeth for Iraqi and Yemeni students by age and sex. For Sinjar students males reported higher percentage for all age groups than Thamar males with no significant difference between them.

For Sinjar students females also reported higher percentage for all age groups except for 15-16 years old, Yemeni females reported higher percentage $(65 \%)$ but with no significant difference.

In Figure (2) the frequency distribution of severity of dental fluorosis for upper posterior teeth for Iraqi and Yemeni students by age and sex. For Sinjar students males and females reported higher percentages for all age groups than Thamar students males and females with significant difference between them at $P<0.001$.

Figure (3): Described the frequency distribution of severity of dental fluorosis for lower anterior teeth for Iraqi and
Yemeni students by age and sex. For Sinjar students males reported higher percentage than Thamar student males with significant difference at $P<0.001$ for the first three age groups and at $P<0 . .01$ for $16-17$ years old. While for Sinjar students females also reported higher percentages in all age groups with significant difference at $P<0.001$ for $14-15$ years old and at $P<0.05$ for $16-17$ years old, with no significant difference for the other age groups.

Figure (4): Represented the frequency distribution of severity of dental fluorosis of lower posterior teeth for Iraqi and Yemeni students by age and sex.

For Sinjar students males and females reported higher percentages for all age groups than Thamar students males and females with significant difference between them at $P<0.001$ except for females group of 13-14 years old with no significant difference between them. 


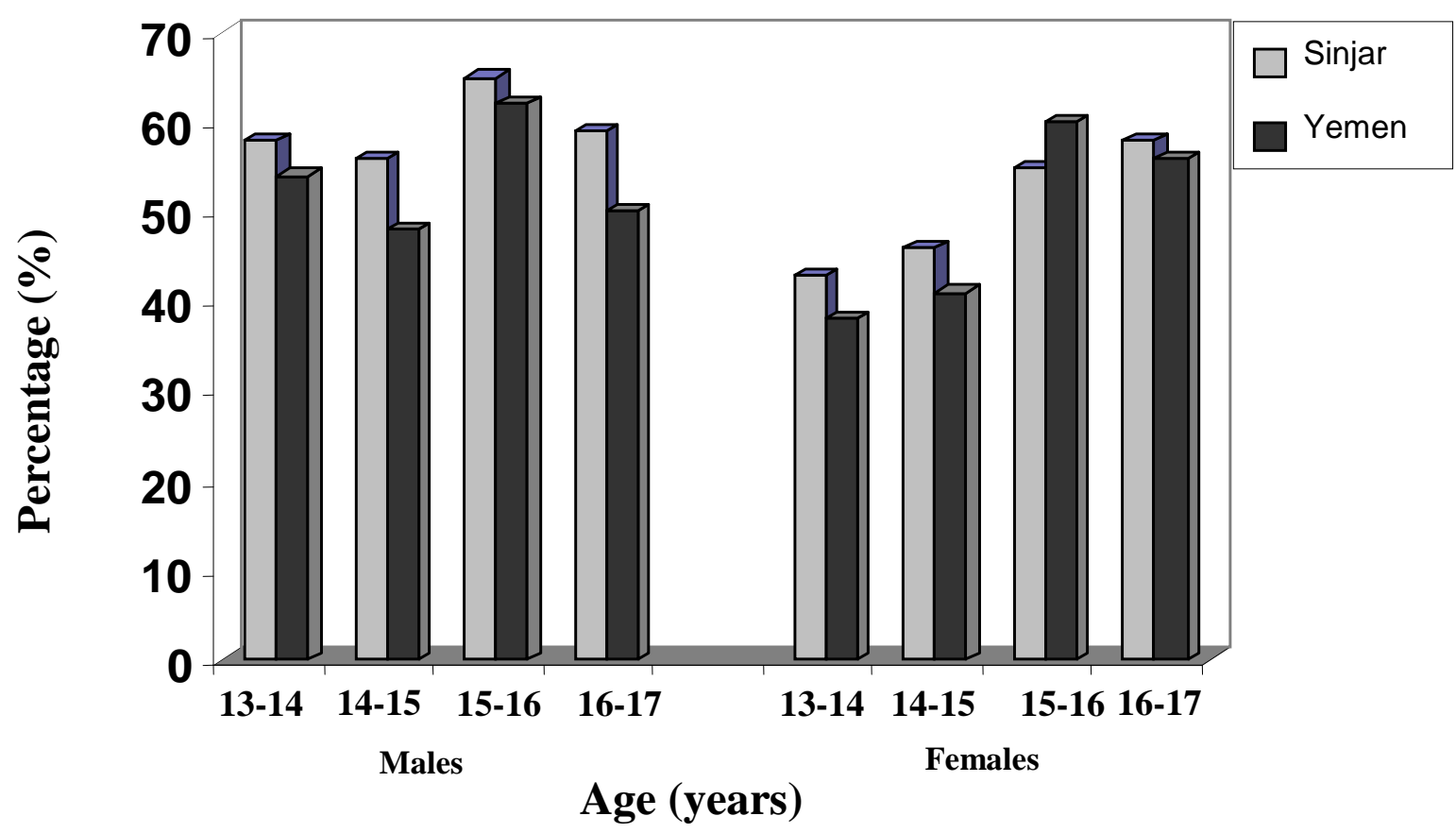

* No significant difference between Iraqis and Yemenis.

Figure (1): Frequency distribution of severity of dental fluorosis for upper anterior teeth for Iraqi and Yemeni students by age and sex.

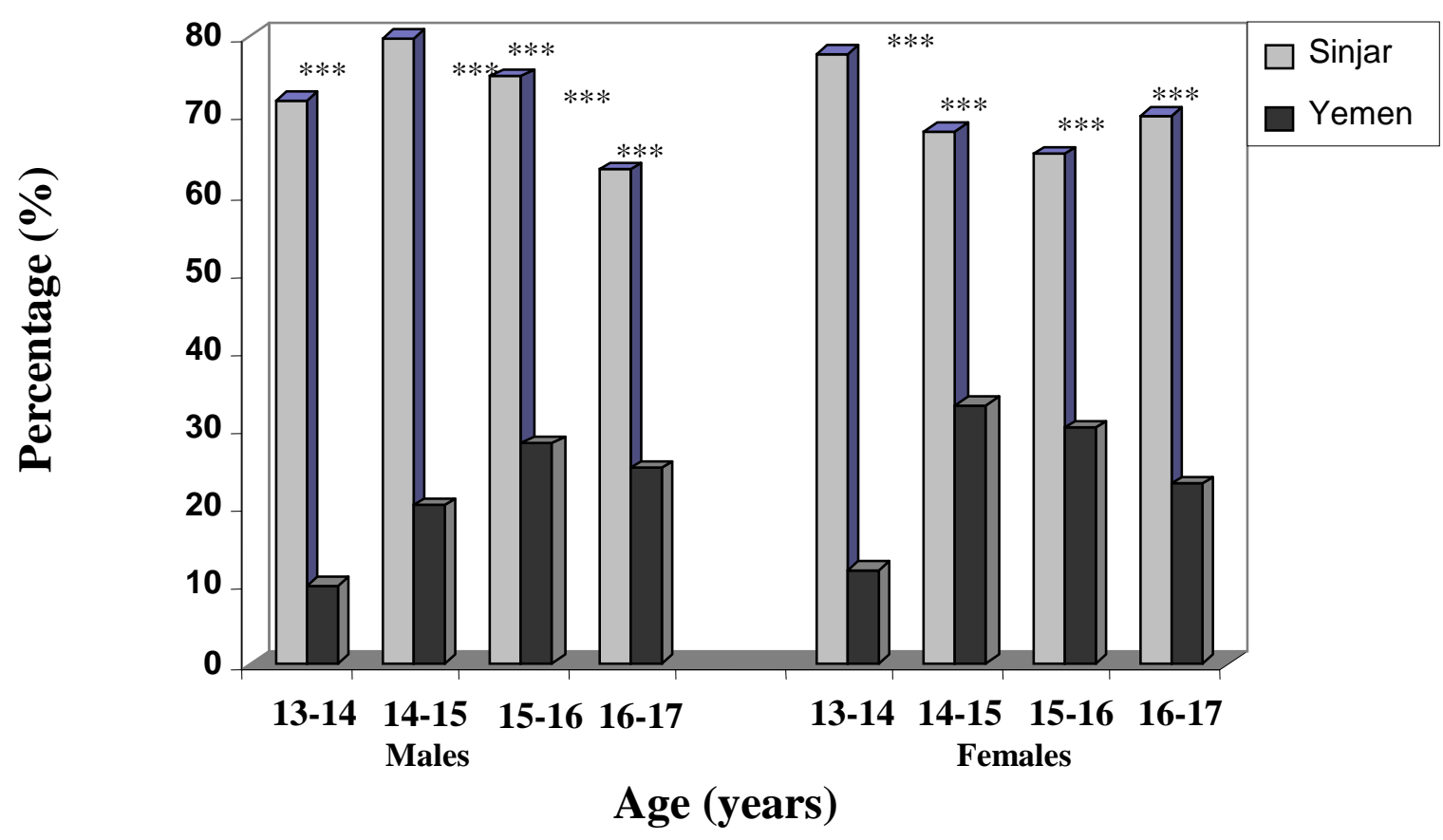

*** Significant difference at $P<0.001$.

Figure (2): Frequency distribution of severity of dental fluorosis for upper posterior teeth for Iraqi and Yemeni students by age and sex. 


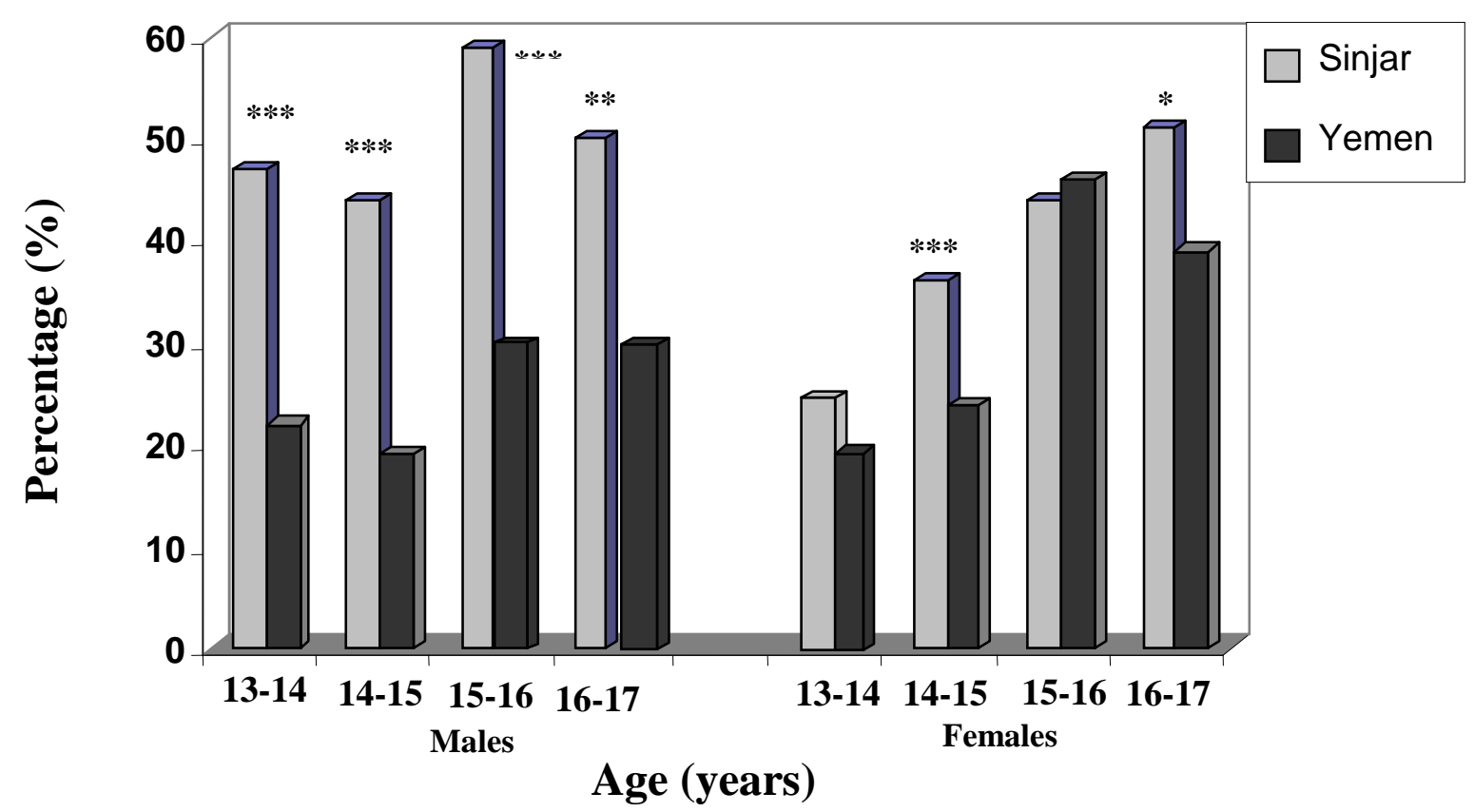

* Significant difference at $P<0.05, * *$ at $P<0.01$ and $* * *$ at $P<0.001$.

Figure (3): Frequency distribution of severity of dental fluorosis for upper lower anterior teeth for Iraqi and Yemeni students by age and sex.

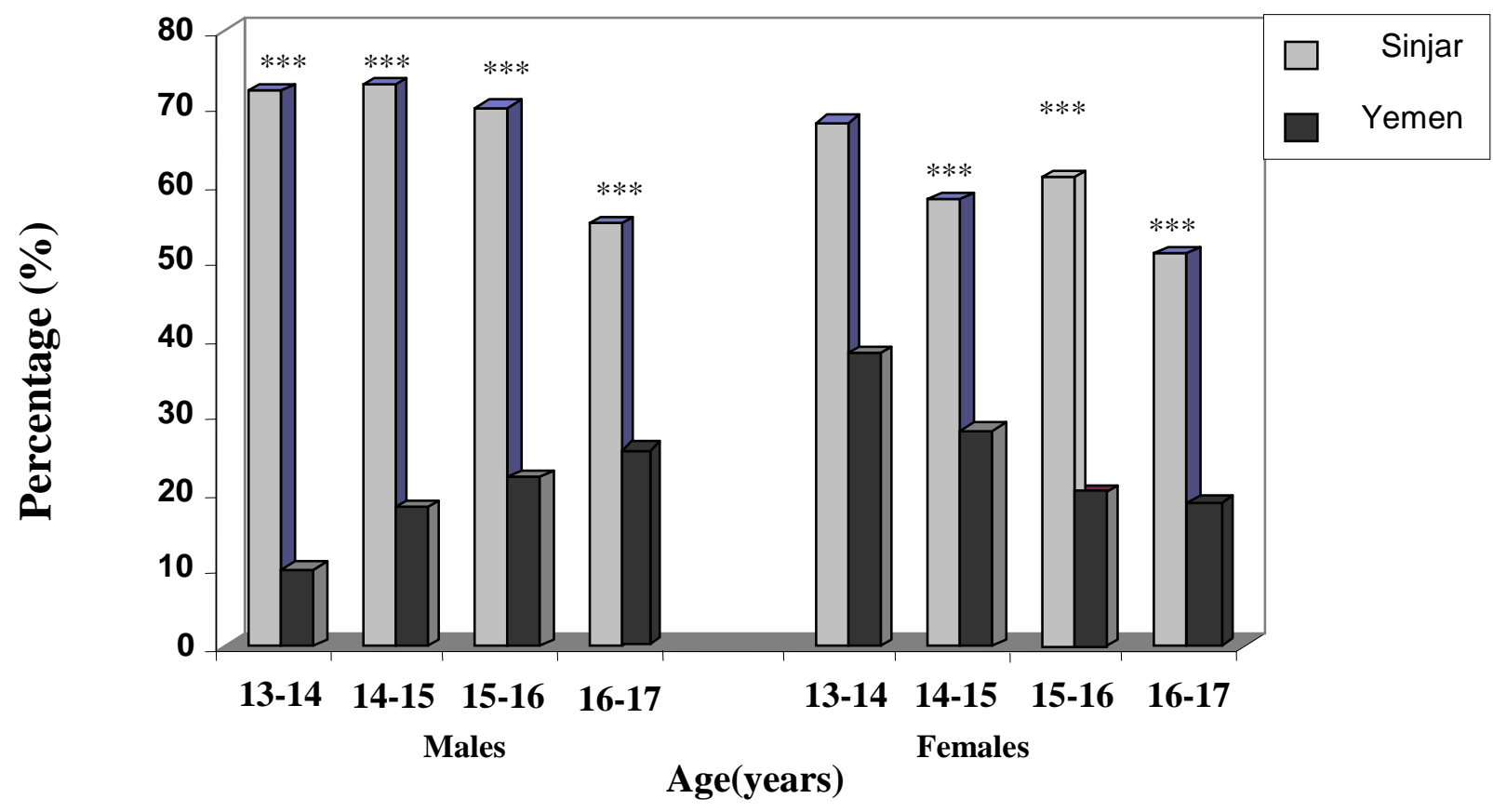

*** Significant difference at $P<0.001$.

Figure (4): Frequency distribution of severity of dental fluorosis for upper lower posterior teeth for Iraqi and Yemeni students by age and sex. 


\section{DISCUSSION}

The presence of natural fluoride in high concentration exceeding the optimal levels will reduce the dental caries 16-18, and unfortunately result in unacceptable appearance of enamel of the teeth, so the study of different concentrations of fluoride and their effects will produce dental fluorosis ${ }^{(19,21)}$.

Dean index was used for assessment of dental fluorosis prevalence within the considered students, in addition to that it had a clear picture about the community fluorosis score which can be calculated easily ${ }^{(13)}$.

Sinjar province was with fluoride drinking water ranges between (2.05-2.22 ppm) which is considered as a high fluoride level area i.e. the fluoride level is twice the recommended optimum level of fluoride. While Thamar province is considered a moderate fluoride level area of drinking water (1.8-2.2ppm) i.e. the fluoride level is twice the recommended optimum level of fluoride.

The results reveled a prevalence of dental fluorosis within Sinjar students equal to $52.1 \%$ for individuals, while in Thamar province it was $16.99 \%$ of individuals that had dental fluorosis. The first percentage was considered as a high prevalence of dental fluorosis, while the second one considered as the lowest. These results were in agreement with other studies in areas which nearly the same level of fluoride in their drinking water for individuals for Sinjar province ${ }^{(22-25)}$, while for Thamar province was in agreement with other studies with nearly the same level of fluoride in their drinking water ${ }^{(24,26,27)}$.

The prevalence of fluorosis for Sinjar student's teeth was $60.53 \%$ while for Thamar student's teeth was $30.23 \%$, i.e. Thamar percentage was nearly half than that of Sinjar which was nearly the same result of other studies ${ }^{(10,11,15)}$.

The prevalence of fluorosis within teeth was higher than for individuals in Sinjar province which had been considered as a high prevalence and it is in contrast with other studies in areas with nearly the same level of fluoride in their drinking water ${ }^{(22-25)}$ and it was nearly the same results of other studies ${ }^{(11,15,28)}$. The high prevalence in Sinjar province may be related to climatic condition which lead to high attitude of the individuals for consumption of high quantity of water specially at high temperature that lead to increase in fluoride concentration reaching the body during teeth formation, while the low prevalence of fluorosis compared with fluoride concentration in drinking water had been found related to the low temperature of climatic condition of Thamar province especially in winter season which lead to the low attitude of the individual for consumption of small quantity of water from bore hole. In addition to that, they drink canned bottled water which lead to decrease in fluoride concentration reaching the body during teeth formation which will affect teeth and create dental fluorosis. Also the difference between examiners in interpretation of criteria of the index used may cause this variation of prevalence in dental fluorosis ${ }^{(20)}$.

In Sinjar the highest percentage of the type of dental fluorosis was questionable, very mild, mild forms which nearly had the same percentages in all age groups and for the total sample, for individuals, and questionable to very mild form for teeth, this gave us the support to finding of many studies ${ }^{(10,11,22,24,29)}$. While in Thamar province showed that the very mild to moderate form for the individual and very mild for teeth and this has given support to findings of many studies $(10,22,23,28,29)$.

The community dental fluorosis index for Sinjar province was 1.62 which is regarded medium according to the public health significance of community fluorosis index scores ${ }^{(30)}$, it was in accordance with the results of other studies ${ }^{(10,22,23,24)}$, but it was in contrast with other studies with nearly the same fluoride concentration in their drinking water, It was higher than that of Dean et al. ${ }^{(31)}$ for Coalsburg city (USA) and that of segretto et al. ${ }^{(23)}$ for both cities (FI-stukton an littke Field) and Forrest ${ }^{(28)}$. This high community fluorosis index for Sinjar province indicated the high consumption of fluoride is mainly from water and increase the effect of fluoride, which was due to climatic condition of province. While the community dental fluorosis index for Thamar province was 3.05 which regarded as very marked according to the public health significance 
of community fluorosis index of scores ${ }^{(30)}$, that is higher than the study of Salman ${ }^{(11)}$ for 6-12 years old primary school children in Thamar which is attributed to the fact of increasing fluorosis with increasing age specially the concentration ranges from 1.8-2.2 ppm which regarded as moderate natural fluoride level, this level was higher than that of Sinjar although the concentration of fluoride in Sinjar was higher than Thamar, that was attributed to the number of individuals in each category of the number of fluorosis index when calculated the community fluorosis index ${ }^{(15)}$.

From the figures it was clear that frequency distribution of severity of fluorosis for Sinjar province is higher than that for Thamar province in all segments of teeth (UA, UP, LA, LP) but with no significant difference for upper anterior and it was with varying degree of significancy for the other segments with the highest percentages in upper and lower posterior teeth $(75 \%, 72 \%)$, and the lowest percentage for the lower anterior teeth (55\%) which was regarded higher than other studies use permanent teeth only which reported $35 \%{ }^{(32)}$ to $39 \%(26,33)$ which was reported by Al-Alousi et al. This high prevalence in this area may be related to the climatic condition of Sinjar province. The high temperature especially hot season (summer) leads to high attitude of the individual for consumption of high quantity of water. This leads to increasing in fluoride concentration reached the body of the students during teeth formation and concentrates on posterior teeth (upper and lower) using higher percentages than other segments of the mouth.

For comparison of severity of dental fluorosis with increasing age of the students, it has been shown that for Sinjar province is higher in all age groups than Thamar province although it was infrequent this result was in contrast with the study of Al-Ajrab et al. and Khamrco et al. ${ }^{(10,34)}$, which reported increasing severity with increasing age and other studies of the same result ${ }^{(20,23,35)}$. The difference between examiners in interpretation of criteria of the index may cause this variation in the prevalence dental fluorosis, while in Thamar the prevalence increased with the increasing age for questionable, very mild, mild forms significantly, this is in contrast with the result of Vincent et al. ${ }^{(19)}$ who found no association between the age of the individual and percent of occurrence of dental fluorosis . For sex difference, females reported less prevalence than males for both provinces for the first three degree of fluorosis this result was in contrast with other studies ${ }^{(19,36,37)}$.

\section{CONCLUSIONS}

From the results of this study, we could easily recognized that there was a great difference observed between the two provinces in prevalence of dental fluorosis and this difference was found to be of high significancy, this is due to the difference in concentration of fluoride in their drinking water supplies.

\section{REFERENCES}

1. Warren JJ, Levy SM. Current and future role of fluoride in nutrition. Dent Clin of North Am. 2003; 47: 224-243.

2. Fomon S, Ekstrand J, Ziegler EE. Fluoride intake and prevalence of dental fluorosis: trends in fluoride intake with special attention to infants. $J$ of Public Health Dent. 2000; 60(3): 131-139.

3. Whelton H, Crowley E, O'mullane D, Donaldson M, Kelleher V, Cronin M. Dental caries and enamel fluorosis among the fluoride and non fluoridated populations in the republic of Ireland in 2002. Community Dent Health. 2004; 21: 37-44.

4. Merrett MC. A blind caries and fluorosis prevalence study of school children in naturally fluoridated an non-fluoridated townships of Morayshire, Scottland. Community Dent Oral Epidemiol. 2002; 30: 70-79.

5. Marshal TA et al. Association between intakes of fluoride from Beverages during infancy an dental fluorosis of primary teeth. $J$ of the Am Coll of Nutr. 2004; 23: 108-116.

6. Zohouri FV, Rugg-Gunn AJ: Sources of dietary fluoride intake in 4-year-ol children residing in low medium and 
high fluoride areas in Iran. Int $J$ Food Sci Nutr. 2000; 51: 317-326.

7. Akapta ES, Fakiha Z, Khann N. Dental fluorosis in $12-15$ years old rural children exposed to fluorosies from well drinking water in the hail region of Saudi Arabia. Community Dent Oral Epidemiol. 1997; 25: 324327.

8. Alarcon-Herrera MT et al. Well water fluoride. Dental fluorosis, Bone fracture in the Guadiana valley of Mexico. Fluoride. 2001; 34(2): 139-149.

9. Peterson G, Kambara M. Remineralisation study of artificial root caries lesions of the fluoride treatment, Gerodontol. 2004; 21(2): 85-92.

10. Al-Ajrab MG . Dental fluorosis and dental caries in Iraq, children living in areas with low an high level of natural water fluoride. 2000; MSc Thesis submitted to the University of Mosul.

11. Salman FD. Prevalence of dental fluorosis among primary school children in Thamar-Yemen. Accepted for publication in $A l-$ Rafidain Dent $J$ in 2006.

12. World Health Organization. Export committee on oral status and fluoride use of fluoride and oral health. Report of a WHO exports committee on oral health status and fluoride use. WHO/ technical report series. 1994; 846.

13. Dean HT. Classification of Mottled enamel diagnosis. J Am Dent Assoc. 1934; 20: 313-319.

14. Dean HT. Production of mottled enamel halted by a change in community water supply. Am J Pub Hlth. 1939; 29: 567-575.

15. Dean H, Arnold, D, Elvove E. Domestic water an dental caries, additional studies of the relation of fluoride domestic water to dental caries experience in 4425 white children age $12-$ 14 years and of 13 cities in 7 states. Public Health Rep. 1942; 87: 11551179.

16. Thylstrup A, Bill J, Brunn B. Caries experience of Danish children living in area with low and optimum levels of natural fluoride. Caries Res. 1982; 166: 413-420.

17. Moola MH. Fluoridation of south Africa. Community Dent Health. 1995;
13(2): 51-55.

18. Khamrco TY, AL-Ajrab MG. Prevalence of dental caries (DMFT) in Iraqi children an adolescents living in areas with low and high levels of natural fluoride. Al-Rafidain Dent J. 2001;1 (sp Iss): 385-400.

19. Vincent A, Segretto D, Charles T. A current study of mottled enamel in Texas. J Am Dent Assoc. 1984; 4: 108-111.

20. Gasper MR, Periera AC, Moreira BH. Estimation of opacities of fluoride origin from fluoride area contained $(0.2 \mathrm{ppm})$ fluoride and optimal (0.7 ppm) concentration. Br Dent J. 1995; 5(2): 13-18.

21. Pereira AC, Moreira BH. Analysis of three dental fluorosis indices used in epidemiological trial. Br Dent J. 1999; 10 (1): 29-37.

22. Driscoll WS, Horowitz HS, Meyers BJ. Prevalence of dental caries and dental fluorosis in areas with optimal and above optimal water fluoride concentrations. J Am Dent Assoc. 1983; 107: 42-47.

23. Segretto VA, Collins FM, Camman D, Smith CT. A current study of mottled enamel in Texas. $J$ Am Dent Assoc. 1984; 113: 29-33.

24. Al-Khateeb TL, Darwish SK, Bastawi AE, O'Mullane DU. Dental caries in fluoride in the drinking water. Community Dent Health. 1990; 7: 165-171.

25. Riordan P, Bank J. Dental fluorosis and fluoride exposure in Western Australia. J Dent Res. 1991; 70: 1072-82.

26. AL-Alousi W. Enamel mottling in Iraqi young adult (an epidemiological study) Iraqi Dent J. 1998; 23: 55-66.

27. Ellwood RP, O'Mullane DU. The demographic and social variation in the prevalence of dental enamel opacities in North Wales. Community Dent Health. 1994; 11: 192-196.

28. Forrest JR. Caries evidences and enamel defect in area with different levels of fluoride in the drinking water. $B r$. Dent J. 1956, 100(8): 195-200.

29. Moller JJ, Pindborg JJ, Roed PB. The prevalence of dental fluorosis in the people of Uganda. Archs Oral Biol. 1970; 19: 213-225. 
30. WHO. Oral Health Surveys. Basic Methods. $4^{\text {th }}$ E. World Health Organization, Geneva, Switzerland. 1997.

31. Dean H, Jay P, Arnold F, Elvove E. Domestic waters and dental caries II: a study of 2832 white children ages 1214 years of eight sub-urban Chicago communities including lactobacillus acidophilus studies of 1761 children. Public Health Rep. 1941; 56: 761792.

32. Jackson D, James P, Wolfe W. Fluoridation in Anglesey. Br Dent $J$. 1975; 138: 165-171.

33. AL-Alousi W, Jackson D, Crompton $\mathrm{G}$, Jenkins O. Enamel mottling in a fluoride and a non fluoride community. Br Dent J. 1975; 138: 9-15.
34. Khamrco TY, AL-Ajrab MG. Naturally fluoride contained of drinking water and the prevalence of dental fluorosis in Ninevah Governorate, Iraq. $A l-$ Rafidain Dent J. 2002; 1: 7-19.

35. Kailis DG, Silva D. Occurrence of dental fluorosis in carnarron Western Australia. Aust. Dent J. 1970; 15: 3543.

36. Galagan DJ, Lamson GG. Climate and endemic dental fluorosis. Pub Hlth Rep. 1953; 68: 497-508.

37. Leverett DH. Prevalence of dental fluorosis in fluoridated and nonfluoridated communities A preliminary investigation. $J$ Public Health Dent. 1986; 46: 184-187. 